\title{
Clinical Significance of Preoperative Embolization for Non-Hypervascular Metastatic Spine Tumors
}

\author{
Sung-Lim Yoo, M.D., ${ }^{1}$ Young-Hoon Kim, M.D., ${ }^{1}$ Hyung-Youl Park, M.D., ${ }^{1, *}$ Sang-Il Kim, M.D., ${ }^{1, *}$ Kee-Yong Ha, M.D., \\ Hyung-Ki Min, M.D., Jun-Yeong Seo, M.D., ${ }^{2}$ In-Soo Oh, M.D., ${ }^{3}$ Dong-Gune Chang, M.D., ${ }^{4}$ Joo-Hyun Ahn, M.D., ${ }^{1}$ \\ Yong-Woo Kim, M.D.' \\ Department of Orthopedic Surgery, Seoul St. Mary's Hospital, College of Medicine, The Catholic University of Korea, Seoul, Korea \\ Department of Orthopedic Surgery, ${ }^{2}$ Jeju National University Hospital, Jeju National University School of Medicine, Jeju, Korea \\ Department of Orthopedic Surgery, Incheon St. Mary's Hospital, College of Medicine, The Catholic University of Korea, Incheon, Korea \\ Department of Orthopedic Surgery, ${ }^{4}$ Sanggye Paik Hospital, The Inje University College of Medicine, Seoul, Korea
}

Objective : The efficacy of preoperative embolization for hypervascular metastatic spine disease (MSD) such as renal cell and thyroid cancers has been reported. However, the debate on the efficacy of preoperative embolization for non-hypervascular MSD still remains unsettled. The purpose of this study is to determine whether preoperative embolization for non-hypervascular MSD decreases perioperative blood loss.

Methods : A total of 79 patients (36 cases of preoperative embolization and 43 cases of non-embolization) who underwent surgery for metastatic spine lesions were included. Representative hypervascular tumors such as renal cell and thyroid cancers were excluded. Intraoperative and perioperative estimated blood losses (EBL), total number of transfusion and calibrated EBL were recorded in the embolization and non-embolization groups. The differences in EBL were also compared along with the type of surgery. In addition, the incidence of Adamkiewicz artery and complications of embolization were assessed.

Results : The average age of 50 males and 29 females was $57.6 \pm 13.5$ years. Lung (30), hepatocellular (14), gastrointestinal (nine) and others (26) were the primary cancers. The demographic data was not significantly different between the embolization and the non-embolization groups. There were no significant differences in intraoperative EBL, perioperative EBL, total transfusion and calibrated EBL between two groups. However, intraoperative EBL and total transfusion in patients with preoperative embolization were significantly lower than in non-embolization in the corpectomy group (1645.5 vs. $892.6 \mathrm{~mL}, p=0.017$ for intraoperative EBL and 6.1 vs. 3.9, $p=0.018$ for number of transfusion). In addition, the presence of Adamkiewicz artery at the index level was noted in two patients. Disruption of this major feeder artery resulted in significant changes in intraoperative neuromonitoring.

Conclusion : Preoperative embolization for non-hypervascular MSD did not reduce perioperative blood loss. However, the embolization significantly reduced intraoperative bleeding and total transfusion in corpectomy group. Moreover, the procedure provided insights into the anatomy of tumor and spinal cord vasculature.

Key Words : Neoplasm metastasis · Spine $\cdot$ Embolization therapeutic $\cdot$ Postoperative hemorrhage $\cdot$ Complications.

- Received : March 31, 2018 •Revised : May 13, 2018 •Accepted : June 19, 2018

- Address for reprints : Young-Hoon Kim, M.D.

Department of Orthopedic Surgery, Seoul St. Mary's Hospital, College of Medicine, The Catholic University of Korea, 222 Banpo-daero, Seocho-gu, Seoul 06591, Korea Tel : +82-2-2258-2837, Fax : +82-2-535-9834, E-mail : boscoa@catholic.ac.kr

* Hyung-Youl Park and Sang-II Kim equally contributed to this study.

This is an Open Access article distributed under the terms of the Creative Commons Attribution Non-Commercial License (http://creativecommons.org/licenses/by-nc/4.0) which permits unrestricted non-commercial use, distribution, and reproduction in any medium, provided the original work is properly cited. 


\section{INTRODUCTION}

The incidence of metastatic spine disease (MSD) is increasing due to improved survival of cancer patients. Surgical interventions for MSD have been increasingly performed, because MSD impairs the quality of life in terms of severe pain, pathologic fractures and risk of paraplegia, ${ }^{5,716,19,20,22)}$. However, spinal surgeries such as total en-bloc spondylectomy or corpectomy may cause severe perioperative bleeding resulting in postoperative complications and poor surgical outcomes. Moreover, highly vascularized tumors including renal and thyroid carcinoma are associated with a high risk of massive blood loss. In this regard, transarterial embolization has been reported to decrease perioperative bleeding in hypervascular tumors $^{5,8-10,12,15,16,21)}$. However, consensus regarding embolization in non-hypervascular spinal metastasis has yet to be achieved $^{3,8,16,17)}$

In addition to reduced perioperative bleeding, preoperative embolization has another advantage in evaluating the vascularity of the lesion and spinal cord ${ }^{19)}$. However, simultaneous embolization and extensive surgery for MSD increase the risk of spinal cord injury and impairment although vascular insult following major spinal surgery has rarely been reported ${ }^{2)}$. Therefore, the purpose of this study was to evaluate the efficacy of preoperative transarterial embolization in non-hypervascular MSD and the vascular safety of the spinal cord.

\section{METHODS AND METHODS}

\section{Patients and surgical procedures}

A clinical and radiological database from 2011 to 2016 was retrospectively reviewed at a single institution. This study was approved by the Institutional Review Board of Seoul St. Mary's Hosptial (IRB approval No. KC15RISE0947). We included patients with the following criteria : 1) adult patients diagnosed with metastatic spine lesion and 2) treatment with reconstructive surgery. The exclusion criteria were : 1) metastatic spine lesion with highly vascular tumor such as renal or thyroid carcinoma and 2) combined surgery for non-spinal metastatic tumors. One hundred eighty-two patients who underwent spinal surgery for MSD were screened and a total of 79 consecutive patients who met these criteria were included in this study. Nine patients were excluded because of known highly vascular tumors (three thyroid and six renal carcinomas). Ninety-four patients who underwent non-reconstruction surgery such as percutaneous vertebroplasty and kyphoplasty were also excluded. General condition of the patients (revised Tokuhashi score) ${ }^{18)}$ and instability of the spine (spinal instability neoplastic score, SINS) $)^{4)}$ were used to determine the surgical intervention, as well as location of the main lesions and history of radiation therapy. Three types of surgical intervention were performed : 1) posterior palliative laminectomy and excision of epidural extension of tumors with posterior reconstruction $(n=41), 2)$ posterior vertebral column resection with posterior reconstruction $(\mathrm{n}=22)$, and 3 ) anterior corpectomy with anterior or posterior reconstruction $(\mathrm{n}=16)$.

\section{Preoperative embolization}

Pre-operative embolization was determined randomly considering the general condition and emergent surgery due to neurological status. Preoperative embolization was performed in 36 patients (45.6\%). All embolization procedures were conducted within 48 hours prior to surgery. The embolization of the index segmental artery included the cephalad and caudal segmental arteries. Embolization was not performed if there was evidence of major blood supply to the spinal cord (the Adamkiewicz artery). In this series, two patients presented with major blood supply for the anterior spinal artery through the Adamkiewicz artery at the index level. A mechanical coil (Tornado ${ }^{\circledR}$ or Vortx ${ }^{\circledR}$; Cook Medical, Bloomington, IN, USA) was used in most patients.

\section{Estimated blood loss}

Intraoperative (measured by the amount of suction drainage and soaked by gauze) and perioperative (the amount of drain for postoperative 2 days) estimated blood losses (EBLs) were recorded. Calibrated EBL was also computed using the following formula : (preoperative hemoglobin - hemoglobin on postoperative 2 nd day) + total number of transfused packed red blood cell (PRC). One unit of PRC was considered to increase the hemoglobin rate by $1 \mathrm{~g} / \mathrm{dL}$.

\section{Statistical analysis}

Perioperative continuous variables in different groups presented with mean values and standard deviation were compared using Student's t-test. Categorical variables were compared using Pearson's chi-squared test. All statistical analysis 
was performed using SPSS software (IBM SPSS Statistics for Windows, version 24.0; IBM Corp., Armonk, NY, USA). The significance level was set at $p$ value less than 0.05 .

\section{RESULTS}

\section{Patients}

The mean age of the patients was $57.6 \pm 13.5$ years (range, 21-84). Fifty patients were male and 29 patients were female. Lung (30, 38.0\%), hepatocellular (14, 17.7\%), gastrointestinal $(9,11.4 \%)$ and others including hematologic malignancy, breast, uterus, bladder, adrenal gland, hypopharynx, nasopharynx, tongue $(26,32.9 \%)$ were the primary sites of cancer. The lesion locations were : five cervical, 53 thoracic and 21 lumbosacral spines. The mean number of instrumented levels was 3.9 \pm 1.5 . Demographic data such as age, sex, number of instrumented levels, bone mineral density and American society of anesthesiologists physical status classification were not significantly different between the two groups (Table 1). The revised Tokuhashi score and SINS were also not significantly different (7.9 \pm 2.5 vs. $6.5 \pm 2.2, p=0.111$ for Tokuhashi score; $10.4 \pm 2.6$ vs. $10.1 \pm 3.3, p=0.815$ for SINS).

\section{Embolization vs. non-embolization}

Table 2 shows the operation time, intraoperative EBL, perioperative EBL, total number of transfusion and calibrated EBL in the embolization and the non-embolization groups. The operation time was longer in embolization group without significance ( $219.9 \pm 77.7$ vs. $231.9 \pm 79.9$ minutes, $p=0.501)$. The

Table 1. Demographics of the patients between the two groups

\begin{tabular}{|c|c|c|c|}
\hline & Non-embolization $(n=43)$ & Embolization $(n=36)$ & $p$-value \\
\hline Age (years) & $58.7 \pm 13.4$ & $56.1 \pm 13.6$ & 0.395 \\
\hline $\operatorname{Sex}(M: F)$ & $30: 13$ & $20: 16$ & 0.192 \\
\hline \multicolumn{4}{|l|}{ Primary } \\
\hline Lung & 16 & 14 & \\
\hline $\mathrm{HCC}$ & 7 & 7 & \\
\hline Gl & 5 & 4 & \\
\hline Others* & 15 & 11 & \\
\hline Number of instrumented levels & $4.1 \pm 1.5$ & $3.7 \pm 1.3$ & 0.177 \\
\hline BMD (T-score) & $-2.5 \pm 1.1$ & $-2.3 \pm 1.4$ & 0.623 \\
\hline ASA grade $(1,2: 3,4)$ & $36: 7$ & $29: 7$ & 0.714 \\
\hline Revised Tokuhashi score & $7.9 \pm 2.5$ & $6.5 \pm 2.2$ & 0.111 \\
\hline SINS & $10.4 \pm 2.6$ & $10.1 \pm 3.3$ & 0.815 \\
\hline
\end{tabular}

Values are presented as mean \pm standard deviation unless otherwise indicated. ${ }^{*}$ Others including hematologic malignancy, breast, uterus, bladder, adrenal gland, hypopharynx, nasopharynx, tongue cancer. $\mathrm{M}$ : male, F : female, HCC : hepatocellular cancer, GI : gastrointestinal, BMD : bone mineral density, ASA : American Society Anesthesiologists physical status classification, SINS : spinal instability neoplastic score

Table 2. Operation time and estimated blood loss between embolization and non-embolization groups

\begin{tabular}{|c|c|c|c|}
\hline & Non-embolization $(n=43)$ & Embolization $(n=36)$ & $p$-value \\
\hline Operation time & $219.9 \pm 77.7$ & $231.9 \pm 79.9$ & 0.501 \\
\hline Intraoperative EBL (mL) & $1069.8 \pm 869.7$ & $862.5 \pm 526.2$ & 0.215 \\
\hline Perioperative EBL (mL) & $529.0 \pm 287.3$ & $513.9 \pm 415.6$ & 0.849 \\
\hline No. of transfused PRC & $4.1 \pm 2.8$ & $3.7 \pm 2.2$ & 0.540 \\
\hline Cal. EBL* $(\mathrm{g} / \mathrm{dL})$ & $5.5 \pm 3.1$ & $4.9 \pm 3.3$ & 0.420 \\
\hline
\end{tabular}

Values are presented as mean \pm standard deviation. ${ }^{*}$ (preoperative hemoglobin - hemoglobin on postoperative 2 nd day) + total number of transfused red blood cell (PRC). EBL : estimated blood loss, PRC : packed red blood cells, Cal. EBL : calibrated estimated blood loss 
intraoperative EBL and perioperative EBL were not significantly different between the two groups (1069.8 \pm 869.7 vs. $862.5 \pm 526.2 \mathrm{~mL}, p=0.215$ for intraoperative EBL; $529.0 \pm 287.3$ vs. $513.9 \pm 415.6 \mathrm{~mL}, p=0.849$ for perioperative EBL). The total number of transfusion and calibrated EBL were also similar in both groups ( $4.1 \pm 2.8$ vs. $3.7 \pm 2.2, p=0.540$ for total transfusion; $5.5 \pm 3.1$ vs. $4.9 \pm 3.3, p=0.420$ for calibrated $\mathrm{EBL}$ ).

The EBL was compared according to the type of surgery. In patients who had undergone corpectomy, the operation time, perioperative EBL and calibrated EBL were not significantly different between the two groups. However, intraoperative EBL was significantly less in the embolization group compared with the non-embolization group (892.6 \pm 589.0 vs. $1645.5 \pm 1277.8 \mathrm{~mL}, p=0.017)$. Furthermore, the total number of transfusion was also significantly lower in the embolization group than in the non-embolization group (3.9 \pm 2.2 vs. $6.1 \pm$ $2.9, p=0.018)$. In laminectomy group, there were no significant differences in operation time, intraoperative EBL, perioperative EBL, the total number of transfusion and calibrated EBL (Table 3).

\section{Case illustrations : clinical significance of the ar- tery of Adamkiewicz}

Any complications directly related to preoperative embolization were not detected in patients. However, the Adamkiewicz artery was noted in two patients with thoracolumbar lesions. Disruption of this major feeder artery during surgery resulted in significant changes in intraoperative neuromonitoring.

Table 3. The effect of embolization on operation time and estimated blood loss according to type of surgery

\begin{tabular}{|c|c|c|c|c|c|c|}
\hline & \multicolumn{3}{|c|}{ Corpectomy $(n=38)$} & \multicolumn{3}{|c|}{ Laminectomy $(n=41)$} \\
\hline & $\begin{array}{c}\text { Non-embol } \\
(n=11)\end{array}$ & $\begin{array}{l}\text { Embol } \\
(n=27)\end{array}$ & $p$-value & $\begin{array}{l}\text { Non-embol } \\
(n=32)\end{array}$ & $\begin{array}{l}\text { Embol } \\
(n=9)\end{array}$ & $p$-value \\
\hline Operation time & $276.3 \pm 89.3$ & & & $200.5 \pm 63.9$ & $196.0 \pm 24.5$ & 0.838 \\
\hline Intraoperative EBL (mL) & $1645.5 \pm 1277.8$ & $892.6 \pm 589.0$ & 0.017 & $871.9 \pm 583.1$ & $772.2 \pm 268.2$ & 0.624 \\
\hline Perioperative EBL (mL) & $569.7 \pm 378.5$ & $555.3 \pm 454.4$ & 0.927 & $515.0 \pm 254.6$ & $389.6 \pm 247.7$ & 0.197 \\
\hline No. of transfused PRC & $6.1 \pm 2.9$ & $3.9 \pm 2.2$ & 0.018 & $3.3 \pm 2.4$ & $3.0 \pm 2.2$ & 0.698 \\
\hline Cal. EBL ${ }^{*}(\mathrm{~g} / \mathrm{dL})$ & $7.5 \pm 3.4$ & $5.5 \pm 3.5$ & 0.117 & $4.8 \pm 2.7$ & $3.1 \pm 1.6$ & 0.084 \\
\hline
\end{tabular}

Values are presented as mean \pm standard deviation. *(preoperative hemoglobin - hemoglobin on postoperative 2nd day) + total number of transfused red blood cell (PRC). Embol : embolization, EBL : estimated blood loss, PRC : packed red blood cells, Cal. EBL : calibrated estimated blood loss
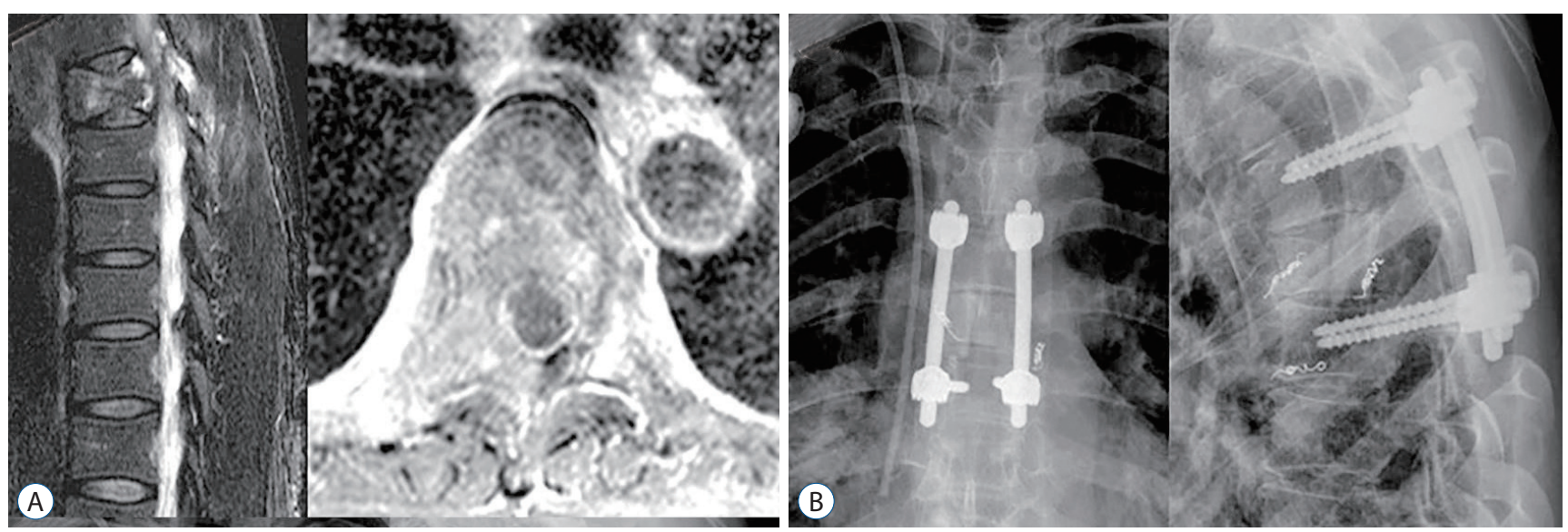

Fig. 1. A case of metastatic spine disease from lung cancer. A : A 58-year-old female patient with lung cancer presented with dorsal back pain and impending cord compression sign. Preoperative sagittal and axial magnetic resonance images showed metastatic lesions resulting in instability and spinal cord compression at T6. B : Palliative surgery (T6 corpectomy, T5-7 anterior interbody graft with posterior instrumentation and posterior fusion) was carried out after preoperative embolization. During the operation, the left side 6 th intercostal artery was clamped. It resulted in loss of MEP in intraoperative neuromonitoring (see also Supplementary Video 1). 


\section{Case 1}

A 58-year-old woman presented with severe back pain due to spinal cord compression with T6 metastasis from lung carcinoma (Tokuhashi score, 10; SINS, 8). Thoracic aortography and intercostal arteriography showed extensive vascularization of the tumor and the major feeder artery from the left side 6th intercostal artery. Both the 7th and right 6th intercostal arteries supplying the tumor were unevenly embolized. Palliative surgery (T6 corpectomy, and T5-7 anterior interbody graft with allogenous fibular bone graft combined with posterior instrumentation and posterior fusion) was performed. The artery of Adamkiewicz was identified on the left
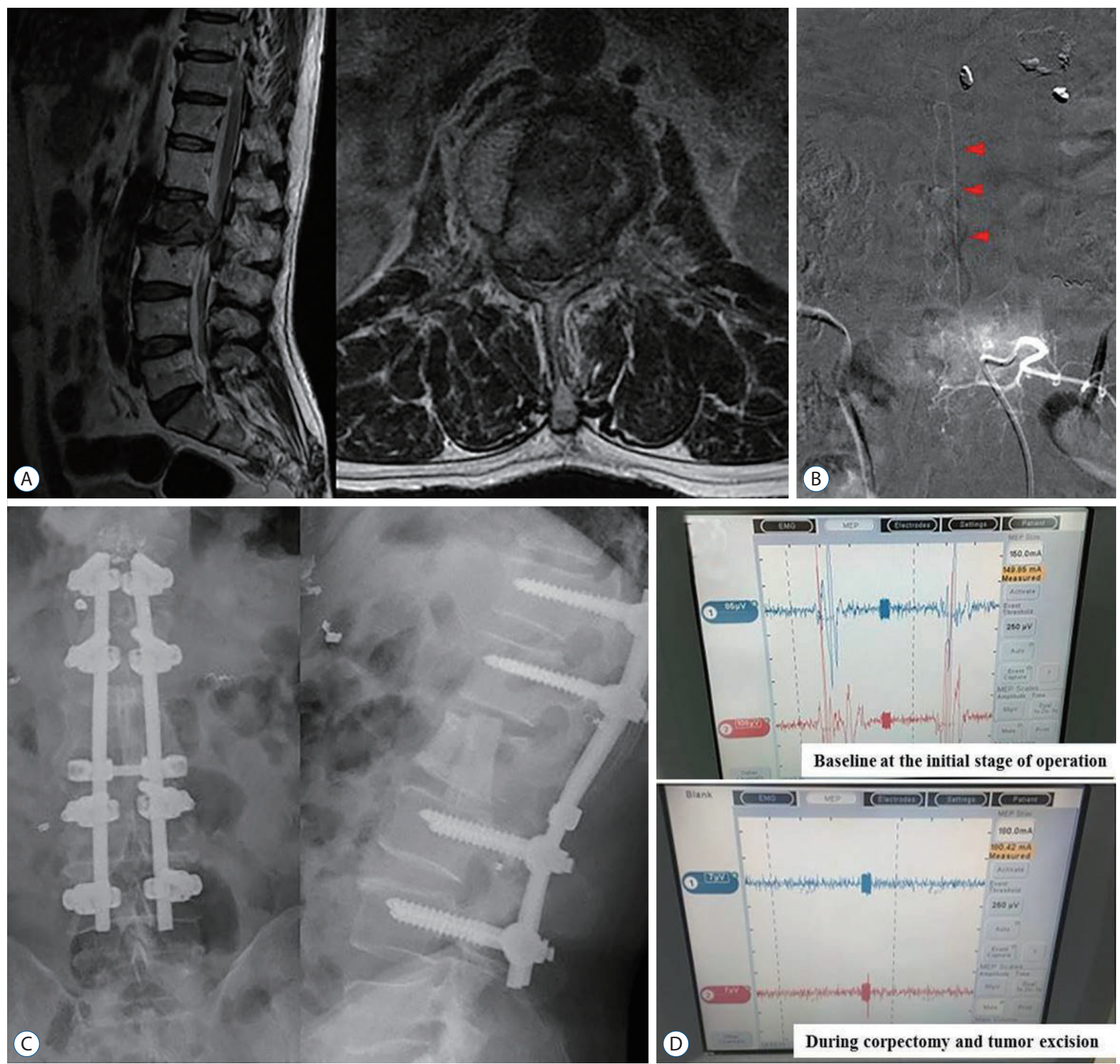

Fig. 2. A case of metastatic spine disease from hepatocellular carcinoma. A : A 61-year-old male with hepatocellular carcinoma presented with progressive L2 metastatic lesions even after radiotherapy. B : Arteriography during preoperative embolization showed the presence of Adamkiewicz artery arising from the left side of $L 2$ segmental artery. Arrowheads indicate the characteristic hairpin turn of the Adamkiewicz artery. $C$ : Palliative surgery (L2 corpectomy, L1-3 anterior interbody graft with posterior instrumentation and posterolateral fusion) was performed. D : However, loss of motor evoked potential in intraoperative neuromonitoring was noted during the excision of tumor and L2 vertebral body. 
6th intercostal artery and temporary clipping showed significant changes on the motor evoked potentials (MEPs) in intraoperative neuromonitoring (Fig. 1, Supplementary Video 1). No postoperative complications or neurological compromise were detected.

\section{Case 2}

A 61-year-old male patient was diagnosed with hepatocellular carcinoma, which metastasized at L2. The patient presented with intractable back pain radiating to bilateral legs due to compression of the spinal cord at the L2 level even after radiotherapy (Tokuhashi score, 10; SINS, 13). The Adamkiewicz artery originating from the segmental artery supplying the tumor was identified during preoperative angiography. The segmental vessels except the left side at L2 level were successfully embolized. Palliative surgery (L2 corpectomy, and L1-3 anterior interbody graft with allogenous fibular bone graft combined with posterior instrumentation and posterolateral fusion) was performed. Despite attempts to avoid injury to the left segmental artery of the L2 during the procedures, the loss of MEP was noted after corpectomy (Fig. 2). The loss of MEP persisted until the end of the surgery. However, the preoperative complaint was relieved without any neurological deficits.

\section{DISCUSSION}

Debate on the efficacy of preoperative embolization for non-hypervascular MSD still persists. Studies reported a decrease in intraoperative blood loss with preoperative embolization while other studies demonstrated no significant differences in EBL after embolization ${ }^{3,8,16,17)}$. In this study, although intraoperative EBL (862.5 vs. $1069.8 \mathrm{~mL}$ ), perioperative EBL (513.9 vs. $529.0 \mathrm{~mL}$ ), transfusion amount (3.7 vs. 4.1), and calibrated EBL (4.9 vs. 5.5) were less in the embolization group, no significant differences were detected between the two groups. Robial et al. ${ }^{16)}$ reported no significant differences in blood loss and transfusion in breast, lung cancer and metastasis other than renal cell carcinoma. Clausen et al. ${ }^{3)}$ also demonstrated that intraoperative blood loss (618 vs. $735 \mathrm{~mL}, p=0.270$ ) and perioperative blood loss ( 902 vs. $985 \mathrm{~mL}, p=0.505$ ) were not significantly different between the two groups. Previous studies included both hypervascular and non-hypervascular tumors and sub-group analysis of non-hypervascular tumors was performed. In this cohort, highly vascular metastatic tumors such as renal and thyroid cancers were excluded and the efficacy of preoperative transarterial embolization in non-hypervascular MSD was evaluated.

The degree of intraoperative blood loss in spinal metastasis may vary according to the operative procedure ${ }^{8}$. Since this study included both corpectomy and laminectomy, the EBL in the two groups was also compared along with the type of surgery. Preoperative embolization significantly decreased intraoperative EBL ( 892.6 vs. $1645.5 \mathrm{~mL}, p=0.017$ ) and the total number of transfusion (3.9 vs. $6.1, p=0.018$ ) in patients treated with corpectomy. Berkefeld et al. ${ }^{2)}$ reported that preoperative embolization using coils and particles significantly reduced mean blood loss in corpectomy (2379 \pm 1844 vs. $4770 \pm 3299$ $\mathrm{mL}, p=0.05$ ). Robial et al. ${ }^{16)}$ also demonstrated preoperative embolization in major surgeries such as corpectomies and vertebrectomies, while limited in palliative procedures such as decompression in all types of metastasis. Based on this study, patients with non-hypervascular MSD may not necessarily benefit from preoperative embolization. Preoperative embolization can be considered selectively for metastatic spinal lesions requiring extensive surgery such as corpectomy and enbloc spondylectomy.

Preoperative embolization was conducted 2 days before surgery in all patients. Surgery performed within 72 hours of embolization has been shown to decrease perioperative blood loss compared with surgery performed beyond this time peri$\mathrm{od}^{1,6)}$. In addition, any complication was not reported in the 36 patients with preoperative embolization, which was consistent with previous studies reporting that preoperative embolization was a safe procedure with a very low complication rate $(0-1 \%)^{12,21)}$.

The clinical significance of the artery of Adamkiewicz was also interesting in this study. The artery of Adamkiewicz, also known as the great anterior radiculomedullary artery, is the most important feeding artery of the thoracolumbar spinal cord. It supplies the lower two-thirds of the spinal cord via the spinal artery. In spine surgery, sacrificing this artery can potentially result in severe neurological deficits. However, $\mathrm{Mu}-$ rakami et al. ${ }^{11)}$ concluded that interruption of the artery of Adamkiewicz did not interfere with neurological function and even recommended that sacrifice of up to three pairs of segmental arteries including the artery of Adamkiewicz does not disrupt the circulation of the spinal cord, based on their 
study of 180 cases of total en-bloc sponydylectomy. In our study, disruption of the Adamkiewicz artery in two patients carrying thoracolumbar lesions during preoperative embolization led to significant changes in intraoperative neuromonitoring (amplitudes and latency in motor evoked potentials, MEPs), without any neurological deterioration in both patients after the surgery. MEP monitoring responds to loss of spinal cord perfusion by the anterior spinal artery ${ }^{14}$. The changes in the MEPs can be explained by the interruption of blood flow to the anterior two-thirds of the spinal cord supplied by the Adamkiewicz artery, which may not necessarily translate to neurological impairment because of the possible compensatory blood supply to the spinal cord. However, Orchowski et al. ${ }^{13)}$ reported that a small percentage of patients $(0.75 \%)$ had major neurological deficits after ligation of a few segmental vessels on one side. Therefore, it is important to recognize the possibility of neurologic deficits after the vascular compromise of spinal cord during surgery. Preoperative angiography provides insights into the anatomy of major feeder artery of spinal cord.

This study has a few limitations. First, the primary sites of cancer were heterogeneous such as lung, hepatocellular, gastrointestinal cancers. Since the number of MSD treated with the surgery was limited, non-hypervascular tumors other than highly vascular tumors were included in this study to minimize tumor heterogeneity. Second, clinical outcomes such as pain and functional status were not evaluated in this study. However, clinical outcomes may be influenced by uncontrolled factors such as general conditions, activity of tumor or other concurrent metastasis. Third, our study showed that preoperative embolization is effective for corpectomy group. But, bias is thought to exist because it is a retrospective study and it is not randomization.

\section{CONCLUSION}

Preoperative embolization for non-hypervascular MSD other than renal and thyroid cancer failed to reduce perioperative blood loss in this study. However, the embolization significantly decreased intraoperative bleeding and total transfusion in the corpectomy group. Furthermore, the procedure provides anatomic insights for the surgeon to avoid vascular compromise of the spinal cord.

\section{CONFLICTS OF INTEREST}

No potential conflict of interest relevant to this article was reported.

\section{INFORMED CONSENT}

This type of study does not require informed consent.

\section{- Acknowledgements}

This study was supported by Research Fund of Seoul St. Mary's Hospital, The Catholic University of Korea.

\section{- Supplementary materials}

The online-only data supplement is available with this article at https://doi.org/10.3340/jkns.2018.0073.

\section{References}

1. Barton PP, Waneck RE, Karnel FJ, Ritschl P, Kramer J, Lechner GL : Embolization of bone metastases. J Vasc Interv Radiol 7 : 81-88, 1996

2. Berkefeld J, Scale D, Kirchner J, Heinrich T, Kollath J : Hypervascular spinal tumors: influence of the embolization technique on perioperative hemorrhage. AJNR Am J Neuroradiol 20 : 757-763, 1999

3. Clausen C, Dahl B, Frevert SC, Hansen LV, Nielsen MB, Lönn L : Preoperative embolization in surgical treatment of spinal metastases: singleblind, randomized controlled clinical trial of efficacy in decreasing intraoperative blood loss. J Vasc Interv Radiol 26 : 402-412.e1, 2015

4. Fisher CG, DiPaola CP, Ryken TC, Bilsky MH, Shaffrey Cl, Berven SH, et al. : A novel classification system for spinal instability in neoplastic disease: an evidence-based approach and expert consensus from the Spine Oncology Study Group. Spine (Phila Pa 1976) 35 : E1221-E1229, 2010

5. Guzman R, Dubach-Schwizer S, Heini P, Lovblad KO, Kalbermatten D, Schroth $G$, et al. : Preoperative transarterial embolization of vertebral metastases. Eur Spine J 14 : 263-268, 2005

6. Heran MK : Preoperative embolization of spinal metastatic disease: rationale and technical considerations. Semin Musculoskelet Radiol $15: 135-142,2011$

7. Jacobs WB, Perrin RG : Evaluation and treatment of spinal metastases: an overview. Neurosurg Focus 11 : e10, 2001

8. Kato S, Murakami H, Minami T, Demura S, Yoshioka K, Matsui O, et al. : Preoperative embolization significantly decreases intraoperative blood loss during palliative surgery for spinal metastasis. Orthopedics 35 : 
e1389- e1395, 2012

9. Kobayashi K, Ozkan E, Tam A, Ensor J, Wallace MJ, Gupta S : Preoperative embolization of spinal tumors: variables affecting intraoperative blood loss after embolization. Acta Radiol 53 : 935-942, 2012

10. Manke C, Bretschneider T, Lenhart M, Strotzer M, Neumann C, Gmeinwieser J, et al. : Spinal metastases from renal cell carcinoma: effect of preoperative particle embolization on intraoperative blood loss. AJNR

Am J Neuroradiol 22 : 997-1003, 2001

11. Murakami H, Kawahara N, Tomita K, Demura S, Kato S, Yoshioka K : Does interruption of the artery of Adamkiewicz during total en bloc spondylectomy affect neurologic function? Spine (Phila Pa 1976) 35 : E1187-E1192, 2010

12. Nair S, Gobin YP, Leng LZ, Marcus JD, Bilsky M, Laufer I, et al. : Preoperative embolization of hypervascular thoracic, lumbar, and sacral spinal column tumors: technique and outcomes from a single center. Interv Neuroradiol 19 : 377-385, 2013

13. Orchowski J, Bridwell KH, Lenke LG : Neurological deficit from a purely vascular etiology after unilateral vessel ligation during anterior thoracolumbar fusion of the spine. Spine (Phila Pa 1976) 30 : 406-410, 2005

14. Pajewski TN, Arlet V, Phillips LH : Current approach on spinal cord monitoring: the point of view of the neurologist, the anesthesiologist and the spine surgeon. Eur Spine J 16 Suppl 2 : S115-S129, 2007

15. Pikis S, Itshayek E, Barzilay Y, Hasharoni A, Kaplan L, Gomori M, et al. :
Preoperative embolization of hypervascular spinal tumors: current practice and center experience. Neurol Res 36 : 502-509, 2014

16. Robial N, Charles YP, Bogorin I, Godet J, Beaujeux R, Boujan F, et al. : Is preoperative embolization a prerequisite for spinal metastases surgical management? Orthop Traumatol Surg Res 98 : 536-542, 2012

17. Thiex R, Harris MB, Sides C, Bono CM, Frerichs KU : The role of preoperative transarterial embolization in spinal tumors. A large single-center experience. Spine J 13 : 141-149, 2013

18. Tokuhashi Y, Matsuzaki H, Oda H, Oshima M, Ryu J : A revised scoring system for preoperative evaluation of metastatic spine tumor prognosis. Spine (Phila Pa 1976) 30 : 2186-2191, 2005

19. Truumees E, Dodwad SN, Kazmierczak CD : Preoperative embolization in the treatment of spinal metastasis. J AM Acad Orthop Surg 18 : 449-453, 2010

20. White AP, Kwon BK, Lindskog DM, Friedlaender GE, Grauer JN : Metastatic disease of the spine. J AM Acad Orthop Surg 14 : 587-598, 2006

21. Wilson MA, Cooke DL, Ghodke B, Mirza SK : Retrospective analysis of preoperative embolization of spinal tumors. AJNR Am J Neuroradiol $31: 656-660,2010$

22. Wirbel RJ, Roth R, Schulte M, Kramann B, Mutschler W : Preoperative embolization in spinal and pelvic metastases. J Orthop Sci $10: 253$ 257, 2005 